\title{
The Application of Visual Illusion in the Fashion Design
}

\author{
HOU Yunshan \\ Chongqing University of Posts and Telecommunications, Chongqing, China \\ Received: August 9, $2021 \quad$ Accepted: August 15, $2021 \quad$ Published: December 30, 2021
}

To cite this article: HOU Yunshan. (2021). The Application of Visual Illusion in the Fashion Design. Asia-Pacific Journal of Humanities and Social Sciences, 1(4), 072-079, DOI: 10.53789/j. 1653-0465.2021.0104.010

To link to this article: https: //doi. org/10.53789/j. 1653-0465.2021.0104.010

\begin{abstract}
Through various visual phenomena, the visual illusion has crossed the boundary between reality and fantasy and has been applied in many fields. It is a creative design element with a sense of modernity and a good theme for various personality expressions in fashion design. Therefore, this paper tries to show that the broad application of visual illusion can improve the creativity of fashion design and maximize the degree of people's senses through visual communication design works, broaden people's horizons, and promote the diversity of fashion design works. In addition, cases of modern fashion design that use visual illusions are found and analyzed. These cases show that the use of creative fashion design through visual illusion is a good example. The significance of this paper is to examine creative fashion design $\mathrm{u}$ sing the visual characteristics of visual illusion.
\end{abstract}

Keywords: fashion design; Gestalt; visual perception; visual illusion

Notes on contributor: HOU Yunshan holds a master's degree in fashion design. She is a lecturer at Chongqing University of Posts and Telecommunications. Her major research interest lies in art education, design theory, and practice. Her email is Houys@ cqupt.edu.cn.

\section{Introduction}

The study of clothing is an indispensable part of human behavior. Only human beings pack the body with materials other than themselves. Clothing not only protects and decorates the human body is also a means of self -expression. People's demand for clothing is not only limited to the function of warmth but also the pursuit of aesthetic and emotional experience. In recent years, the esthetic response has become a prominent part of the consumption process and a focus in creating designed objects (Casto \& DeLong 2019: 105-131). Psychology is the science of human behavior. Gestalt psychology is a modern Western school of psychology that focuses on visual perception. The study of visual illusions has proven to be an advantageous approach in vision science (Gomez-Villa \& Martín \& Vazquez-Corral, et al. 2020: 156-174). Visual illusion has been the research object of Gestalt psychology since 1912. Gestalt school Studies over the past 110 years have provided important information on Visual Illusions. With the development of fashion, the Visual Perception theory of Gestalt is more and 
more used in clothing design.

In recent years, there has been growing interest in Visual Design. There is a growing body of Fashion Designers who realize the Importance of Visual Illusions. The challenge for designers is to create fashion articles of clothing that are aesthetically appropriate and visually engaging for the target audience. Currently, visual illusion is widely used in many fashion design works.

Although a great deal of research has been conducted on apparel design, relatively little research exists on the application of visual perception in apparel design and even less on the application of visual illusion. Most of these studies have only involved thegarment's shape, color, and material.

This paper attempts to show that the widespread application of visual illusion can improve the creativity of fashion design and could maximize peoples' sense degree through the visual communication design works, expand people's horizons, and promote the diversity of fashion design works.

Clothing communicates a message to the viewer through a visual medium. The reason why clothing would the visual illusion phenomenon exist, in short, is that the human eye can consciously balance what they see, and this balance can lead to the visual illusion. For example, a black background will be brighter, and a white background will be darker. The visual illusion is a phenomenon, and it should be analyzed from the angles of physics and philosophy. Therefore, applying this visual illusion to clothing design works can enhance the interest of the work. This research offers a fresh perspective on fashion design.

\section{Theories and Features of Visual Illusion}

The Gestalt principles were initially formulated more than a century ago by researchers working in visual perception (e.g., von Ehrenfels 1890; Wertheimer 1912) (Gallace \& Spence 2011: 538-561). In his 1923 classical paper, the founder of Gestalt psychology, Max Wertheimer, had proposed that what we see is the simplest, most balanced, and regular organization possible under the circumstances. He called this the Prägnanz principle and attributed it to the tendency of the brain towards equilibrium (Spillmann 2006: 67-92). Gestalt psychology questions regarding the emergence of structure in perceptual experience and the subjective nature of phenomenal awareness (e.g., visual illusions, perceptual switching, context effects) continue to inspire contemporary scientific research, using methods and tools that were not at the Gestaltists' disposal (Wagemans \& Elder \& Kubovy, et al. 2012: 1172-1217). The whole is different from the sum of its parts is the main criterion of Gestalt. Gestalt maintains its perceptual properties regardless of figural transformations. When stimuli are close to each other in space and time, they may be perceptually combined into coherent, salient patterns or wholes. When stimuli occur close to one another in space and time, they may group perceptually into coherent, salient patterns or wholes. Such Gestalts, as they are called, abound in our perceptual world, as when leaves and branches cluster into trees, and when trees merge into forests; when eyes, ears, noses, and mouths configure into faces; when musical notes coalesce into chords and melodies; and when countless dots or pixels blend into a photograph (Pomerantz \& Portillo 2011: 1331-1349). The Gestalt approach challenged the view that vision can be understood from analysing stimulus elements (Spillmann 2006: 67-92).

A visual illusion (VI) is an image stimulus that induces a visual percept that is not consistent with the visual information that can be physically measured in the scene (Gomez-Villa \& Martín et al. 2020: 156-174). The 
Asia-Pacific Journal of Humanities and Social Sciences

visual illusion is the contradictory illusion between our recognition and what we observe. When the observer finds the imbalance between subjectivity and object, vision confusion appears. Human relies on vision, audition, olfaction, gustation and tactile. The eyes are a visual perception organ and the main channel to acquire information. Eyes are the visual acceptor, by which we scene different meanings of graphic, namely the visual illusion (Li 2014: 1306-1310).

\section{The Manifestation of Visual Illusion Infashion Design}

Visual communication is completed by two thinking processes: logical thought and illogical thought. The designer will apply logical thought to analyze and synthesize, creatively design, produce and publish to put the message across. This process must be deduced by rigorous logical thought. Then, in the most important creative design phase, innovative illogical thought factors emerge and dominate, becoming the significant formal expression of originality. The combination of science and art in the design, on the one hand, can effectively and scientifically avoid harmful visual illusions. On the other hand, one can skillfully use visual illusions to broaden designers' design ideas and broaden the road of artistic creation (Li 2014: 1306-1310).

There is no doubt that visual illusions intrigue young and old alike, and the fashion industry draws from this. Moreover, the study of visual illusions has a long tradition in the visual sciences as it provides a powerful tool to gain insights into the mechanisms underlying visual perception (Dickson \& Burtan \& James, et al. 2021).

In the current fashion design, many designs use electronic technology to carry out digital printing or use some other techniques to create a visual illusion of clothing. The wide application of visual illusion improves the vitality and vividness of fashion design works and meets the audience's basic needs for the art and novelty of fashion. Visual illusion has a variety of manifestations in fashion design. We can study the visual effect of clothing from a new angle. In this way, we can deepen our understanding of clothing and give consumers more visual impact works. Clothing can be adequately disguised and modified to the wearer's figure scale through visual illusion. Through the color collocation, line structure, three-dimensional outline of clothing to improve the body defects of the wearer, highlight the purpose of the body advantage.

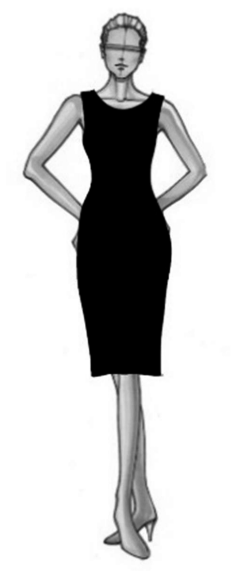

a

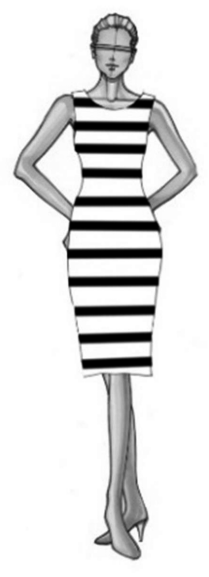

$\mathrm{b}$

Figure 1 Visual Comparison Diagram 


\section{1 Rationalamendment and utilization of visual illusion}

The use of visual illusion to improve visual imaging enhances the aesthetic feeling and body modification, known as the role of positive use. The segmentation of clothing by horizontal stripes and vertical stripes will produce visual illusion, while the segmentation of stripes is a positive use. Through vertical stripes, the audience's eyes can be drawn up or down, respectively, making people wearing clothes look more slender and slender visually. Whether it is two-dimensional graphics, line style, or three-dimensional outline, it can become more diversified under the influence of flexural visual illusion, so that sensory vision becomes more three-dimensional and rich to enhance different clothing design styles. When paying attention to the pattern and shape of clothing, people's visual perception will move with the line direction and structure of clothing pattern under the subconscious influence. People produce visual illusions to some extent. Black has a visual effect, while horizontal stripes have a visual expansion effect. The stylist uses this principle, appropriate use color and line. This will optimize the figure of the wearer. (Figure 1)

\section{2 Correction and improvement of visual illusion}

The visual illusions phenomenon makes objective things become uglier. At this point, the reverse correction can effectively correct, for example, once the visual center does not tally with the geometric object center, dress up and down the size of the local area will make the parts too big or too much, then can improve the garment correct visual illusion at the center of the waistline. The golden ratio of the human body is 5: 8. In real life, most people don't reach the golden ratio. To solve this problem, fashion designers can use the concept of visual illusion to carry out design work - the use of visual illusion in the harmonization and decoration of clothing. The designer guides the transformation of visual perception clues through segmentation illusion so that the height and proportion of the human body can produce a certain range of visual illusions.

\section{Application Method of Visual Illusion in Fashion Design}

\section{1 Horizontal and vertical lines}

Horizontal lines have stability and move left and right to give a sense of peace and vastness to the eyes. When one observes simple plane figures, consisting mainly of only a few lines, often conspicuous differences are manifested between spatial relations as seen directly by the eye and as can be shown indirectly to be present by way of measuring aids (Todorović 2020: 1128-1199). Used in clothing design, it has the visual effect of expansion, can make the body slender or narrow shoulder slightly widened visual illusion feeling. If the horizontal lines are continuously arranged, a horizontal stripe pattern is formed, which does not have the feeling of widening and gives the illusion of lengthening. Therefore, this method is often used on people with thin bodies. (Figure 2)

The illusion of vertical line gives people a sense of straight, so that the track of people's vision moves up and down, creating the illusion of straight and elongated. Accordingly, the dress of vertical line stripe can produce the slender, narrow effect, make up for flaw for the person of fat bodily form, produce an emaciated visual 

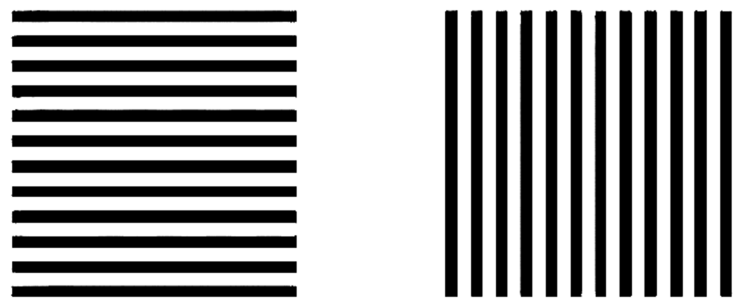

Figure 2 Helmholtz Square Visual Illusion

effect. If the vertical lines are continuously arranged to form a vertical stripe pattern, it will give the impression that people will become wider rather than thinner, so this technique is often used on obese people. (Figure 3 )

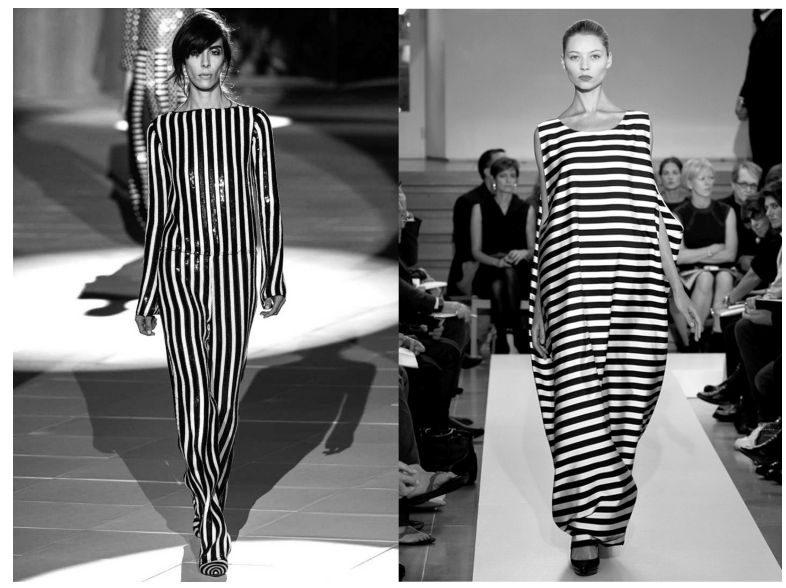

Figure 3 Horizontal and Vertical Lines

\section{2 Sloping lines and curves}

In a scientific American paper on illusions, Gillam (1980) wrote that "Geometrical illusions are line figures in which the length, orientation, curvature or direction of lines is wrongly perceived" (Todorović 2020: 11281199). Slanting lines are visually fluid. It makes people feel unstable. Curves have feminine characteristics. Skillful use of curves can increase the softness of body shape. (Figure 4)

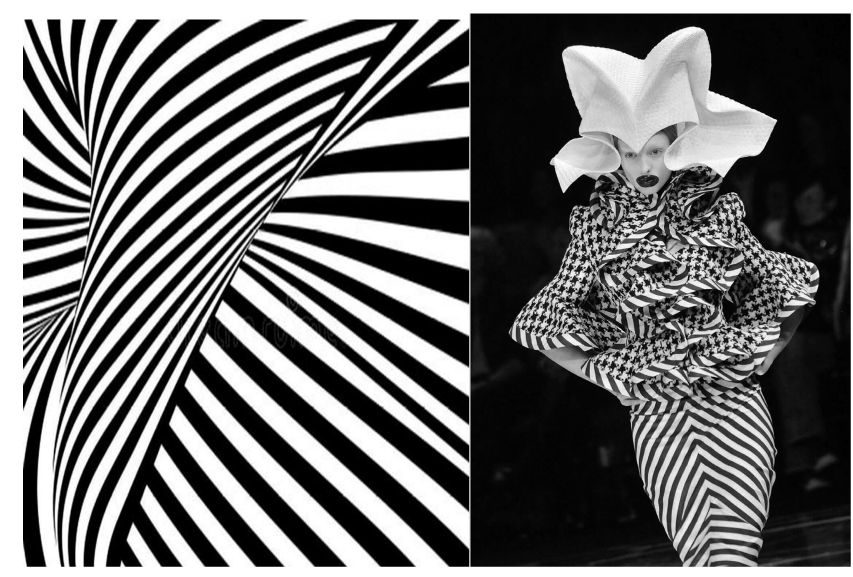

Figure 4 Sloping Lines and Curves 


\section{3 Positive and negative shapes}

Behavioral, neural, and computational considerations suggest that the visual system may use (at least) two approaches to binding an object's features and/or parts into a coherent representation of shape (Hummel 2001: 489-517). Positive and negative shapes, also known as reversed graphics, refer to the interdependence of positive and negative shapes borrowed from each other. In graphic design, the performance of spatial visual illusion, these performances break people's perception of space in life, so that people see space on the plane and expand the visual perception. (Figure 5)
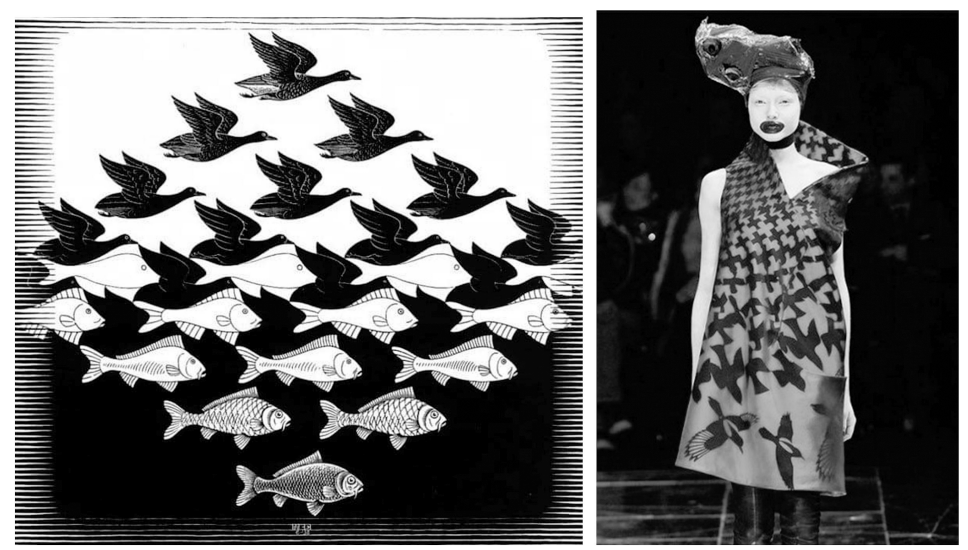

Figure 5 Positive and Negative Forms

\section{4 Color illusions}

Some of the key design elements in visual communications design arecolour and contrast, and these also can enhance the cognitive organisation of incoming visual information as per the Gestalt 'laws' of visual perception. It is perhaps timely to examine the roles of colour and contrast in human visual perception (O'Connor 2015: 85 -92). Color brings visual excitement and emotional communication to people and can produce a certain psychological suggestive effect by stimulating people's retinal nerves. Color is also one of the important elements in pattern design. People's vision will be affected by the color of the surrounding environment and then produce the illusion of color phenomenon. For example, warm colors are heavy, while cold colors are light; warm colors have the feeling of strong density, cold colors have the feeling of thinness. The difference in hue also produces a certain visual illusion. At the same distance, some colors look close, while others look far away, which is the distance illusion on the color. And distance illusion and color wave length and brightness related. Experiments show that the wave length of long brightness high color feeling closer, while the wave length of short brightness low color but feel more distant. (Figure 6)

\section{Conclusion}

This study discusses apparel design activities based on cognitive psychology and visual art design, using the theory of visual illusion as an entry point. Analyzing the concept of visual illusion is not difficult to understand from the literal perspective. Fashion designers use some expression techniques in specific works to express the 


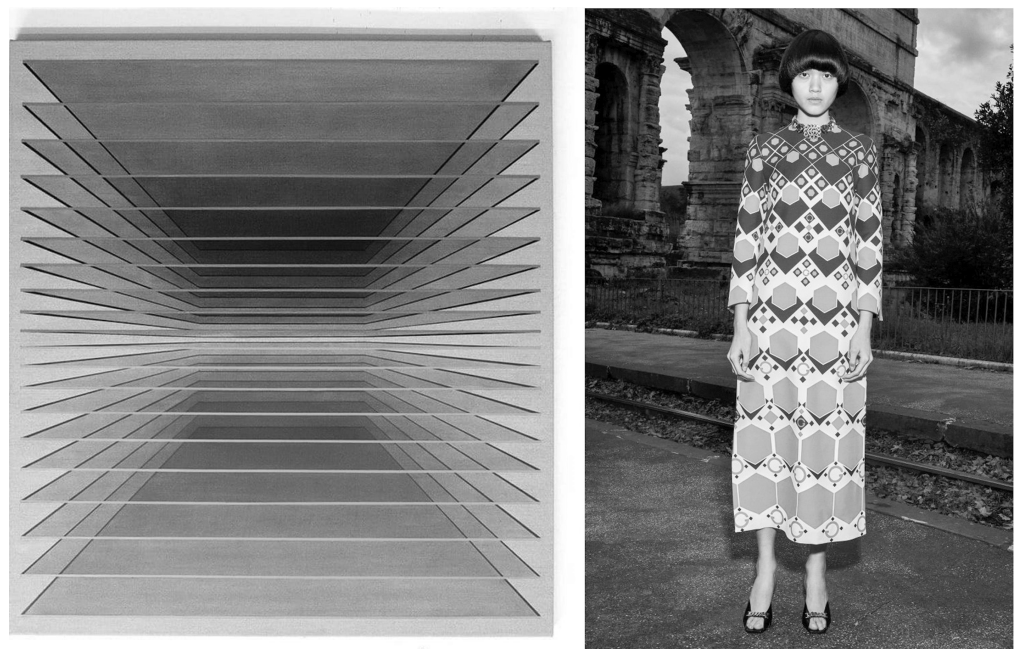

Figure 6 Positive and Negative Forms

shape and pattern of clothing through irregular forms. In clothing, the formation process of visual illusion is a process of "ambiguity of figure itself", which is also an illusion of it. It constructs the relationship between psychology, cognition, visual perception and perceptual output and promotes design research in a multidimensional manner to enrich the research perspectives and practical forms of fashion design. Through the study of the law of visual illusion, designers can be inspired to create the impact of visual illusion on the three-dimensional space of clothing according to the three-dimensional space characteristics of clothing, starting from two perspectives of pattern and texture of clothing, using the visual illusion design of pattern and three-dimensional transformation of fabric to create the effect of visual illusion on the three-dimensional space of clothing.

\section{References}

Casto, M. A. \&Delong, M. (2019). Exploring esthetic response to classic as a means to slow fashion. Fashion Practice, 11, 105 -131 .

Dickson, G., Burtan, D., James, S., Phillips, D., Stevanov, J., Heard, P. \& Leonards, U. (2021). Walking on visual illusions. I-Perception, 12, 204166952098110.

Gallace, A. \& Spence, C. (2011). To what extent do Gestalt grouping principles influence tactile perception? Psychological Bulletin, 137, 538-561.

Gomez-Villa, A., Martín, A., Vazquez-Corral, J., Bertalmío, M. \& Malo, J. (2020). Color illusions also deceive CNNs for low-level vision tasks: analysis and implications. Vision Research, 176, 156-174.

Hummel, J. E. (2001). Complementary solutions to the binding problem in vision: implications for shape perception and object recognition. Visual Cognition, 8, 489-517.

Li, S. (2014). Design and analysis of visual illusion application in visual communication. Applied Mechanics and Materials, 651$653,1306-1310$.

NG Woon Lam. (2021). Dynamic changes of Chinese calligraphy concepts and its simplification in 3D animation space. Asia-Pacific Journal of Humanities and Social Sciences, 1(3), 48-56.

O'Connor, Z. (2015). Colour, contrast and Gestalt theories of perception: the impact in contemporary visual communications design. Color Reseach and Application, 40, 85-92.

Pomerantz, J. R. \& Portillo, M. C. (2011). Grouping and emergent features in vision: toward a theory of basic Gestalts. Journal of Experimental Psychology: Human Perception and Performance, 37, 1331-1349. 
Todorović, D. (2020). What are visual illusions. Perception, 49, 1128-1199.

Wagemans, J., Elder, J. H., Kubovy, M., Palmer, S. E., Peterson, M. A., Singh, M. \& von der Heydt, R. (2012). A century of Gestalt psychology in visual perception: I perceptual grouping and figure-ground organization. Psychological Bulletin, $138,1172-1217$.

ZHANG Pengxia. (2021). Materials and symbols: the primitive cultural form of visual art. Asia-Pacific Journal of Humanities and Social Sciences, 1(1), 113-117.

(Editor: Lucy) 